Published in final edited form as:

Gut. 2015 May ; 64(5): 800-812. doi:10.1136/gutjnl-2014-306996.

\title{
Circulating microRNA signature in non-alcoholic fatty liver disease: from serum non-coding RNAs to liver histology and disease pathogenesis
}

\author{
Carlos J Pirola ${ }^{1}$, Tomas Fernández Gianotti ${ }^{1}$, Gustavo 0 Castaño ${ }^{2}$, Pablo Mallardi ${ }^{3}$, Julio \\ San Martino ${ }^{3}$, María Mora Gonzalez Lopez Ledesma ${ }^{4}$, Diego Flichman ${ }^{4}$, Faridodin \\ Mirshahi $^{5}$, Arun J Sanyal ${ }^{5}$, and Silvia Sookoian ${ }^{2,6}$ \\ ${ }^{1}$ Department of Molecular Genetics and Biology of Complex Diseases, Institute of Medical \\ Research A Lanari-IDIM, University of Buenos Aires-National Scientific and Technical Research \\ Council (CONICET), Ciudad Autónoma de Buenos Aires, Argentina \\ ${ }^{2}$ Liver Unit, Medicine and Surgery Department, Hospital Abel Zubizarreta, Ciudad Autónoma de \\ Buenos Aires, Argentina \\ ${ }^{3}$ Pathology Department, Hospital Diego Thompson, San Martin, Buenos Aires, Argentina \\ ${ }^{4}$ Department of Virology, School of Pharmacy and Biochemistry, University of Buenos Aires, \\ Ciudad Autónoma de Buenos Aires, Argentina \\ ${ }^{5}$ Division of Gastroenterology, Hepatology and Nutrition, Department of Internal Medicine, Virginia \\ Commonwealth University School of Medicine, Richmond, Virginia, USA \\ ${ }^{6}$ Department of Clinical and Molecular Hepatology, Institute of Medical Research A Lanari-IDIM, \\ University of Buenos Aires- National Scientific and Technical Research Council (CONICET), \\ Ciudad Autónoma de Buenos Aires, Argentina
}

\begin{abstract}
Objectives-We used a screening strategy of global serum microRNA (miRNA) profiling, followed by a second stage of independent replication and exploration of liver expression of selected miRNAs to study: (1) the circulating miRNA signature associated with non-alcoholic
\end{abstract}

Copyright Article author (or their employer) 2014.

Correspondence to: Dr Silvia Sookoian, and Dr Carlos J Pirola, Instituto de Investigaciones Medicas, IDIM-CONICET, Combatientes de Malvinas 3150, CABA-1427, Argentina; sookoian.silvia@lanari.fmed.uba.arpirola.carlos@lanari.fmed.uba.ar.

Patient consent Obtained.

Ethics approval Hospital Zubizarreta and Instituto Lanari protocol number: 104/HGAZ/09 and 89/100.

Provenance and peer review Not commissioned; externally peer reviewed.

Contributors CJP: study concept and design; acquisition of data; analysis and interpretation of data; ISH experiment; statistical analysis; drafting of the manuscript; obtained funding; study supervision. TFG: miRNA expression. GOC: performed liver biopsies and collected serum samples. PM and JSM: performed histopathological evaluation and IHQ. MMGLL, DF and TFG: in vitro studies. FM: ISH experiment. AJS: critical revision of the manuscript for important intellectual content; interpretation of ISH results. SS: study concept and design; acquisition of data; ISH experiment; performed liver biopsies and collected biological material; analysis and interpretation of data; drafting of the manuscript; statistical analysis; obtained funding; study supervision.

Competing interests TFG, MMGL, DF, SS and CJP belong to the Consejo Nacional de Investigaciones Científicas y Tecnologicas (CONICET). AJS was also supported by a grant from the NIH RO1 DK 081410. CJP, TGF, GOC, PM, JSM, MMGLL, DF, FM, AJS and SS have no direct competing interests to declare. 
fatty liver disease (NAFLD) progression and predictive power, (2) the role of miRNAs in disease biology and (3) the association between circulating miRNAs and features of the metabolic syndrome.

Methods-The study used a case-control design and included patients with NAFLD proven through biopsy and healthy controls.

Results-Among 84 circulating miRNAs analysed, miR-122, miR-192, miR-19a and miR-19b, miR-125b, and miR-375 were upregulated $>2$-fold $(\mathrm{p}<0.05)$ either in simple steatosis $(\mathrm{SS})$ or nonalcoholic steatohepatitis (NASH). The most dramatic and significant fold changes were observed in the serum levels of miR-122 (7.2-fold change in NASH vs controls and 3.1-fold change in NASH vs SS) and miR-192 (4.4-fold change in NASH vs controls); these results were replicated in the validation set. The majority of serum miR-122 circulate in argonaute2-free forms.

Circulating miR-19a/b and miR-125b were correlated with biomarkers of atherosclerosis. Liver miR-122 expression was 10-fold ( $\mathrm{p}<0.03$ ) downregulated in NASH compared with SS and was preferentially expressed at the edge of lipid-laden hepatocytes. In vitro exploration showed that overexpression of miR-122 enhances alanine aminotransferase activity.

Conclusions-miR-122 plays a role of physiological significance in the biology of NAFLD; circulating miRNAs mirror the histological and molecular events occurring in the liver. NAFLD has a distinguishing circulating miRNA profile associated with a global dysmetabolic disease state and cardiovascular risk.

\section{INTRODUCTION}

MicroRNAs (miRNAs) are small (19-23 nucleotides) non-coding RNA molecules that regulate gene expression acting, at least in part, at a post-transcriptional level and finetuning in concert the activity of thousands of genes and, consequently, their protein synthesis. ${ }^{1}$ Interestingly, miRNAs are stable in clinical samples because they are resistant to the action of ribonucleases; therefore, miRNAs detected in cell-free serum or plasma, also called circulating miRNAs, have been proposed as attractive diagnostic tools for distinguishing, non-invasively, diseased individuals from healthy ones. ${ }^{2}$ The scope of applications associated with miRNAs is even broader because they are used in different clinical settings for early disease detection and monitoring of disease progression and response to treatment for a wide range of disorders.

The increasing enthusiasm in the use of circulating miRNAs in clinical practice is explained by their ability to accurately reflect the physiological/pathological state of the tissue they are derived from. Thus, circulating miRNAs may be regarded as blood-based fingerprints of the affected tissue; therefore, they are powerful tools for the understanding of the disease biology. Finally, circulating miRNAs have a key role as signalling molecules because they are involved in cell-to-cell communication, supporting the idea that they might function as hormones. ${ }^{3-5}$ Consequently, miRNAs might be able to modulate cell phenotypes at distance from the cells they are derived from.

Non-alcoholic fatty liver disease (NAFLD) is a clinical condition that refers to potentially progressive histological changes ranging from fatty liver alone (simple steatosis (SS)) to 
non-alcoholic steatohepatitis (NASH), a disease stage characterised by liver cell injury, mixed inflammatory lobular infiltrate and variable fibrosis. ${ }^{6}$

Although much research is being conducted to decipher the mechanisms associated with disease progression, many issues remain unsolved. For example, there is still missing information about the role of miRNAs in disease biology, and there is no reliable noninvasive disease biomarker for distinguishing mild from severe histological disease stages.

In addition, whereas NAFLD has been implicated in metabolic syndrome (MetSyn)associated phenotypes, such as insulin resistance ${ }^{78}$ and cardiovascular disease (CVD), as reviewed recently, ${ }^{9}$ it is still unknown whether patients with NAFLD have a distinguishing circulating miRNA profile associated with a global dysmetabolic disease state and CVD risk. Hence, we investigated whether circulating miRNAs are able to reflect the physiopathological picture of the affected liver in patients with NAFLD, thus depicting a serum/plasma-based fingerprint of the fatty liver.

For this purpose, we used a screening strategy of global serum miRNA profiling, followed by a second stage of independent replication and exploration of liver expression of selected miRNAs to explore: (1) the circulating miRNA signature associated with NAFLD progression and the diagnostic performance of miRNAs in predicting liver histology, (2) the underlying pathophysiological mechanisms associated with the disease and (3) the association between differential expression of candidate miRNAs and features of the MetSyn.

The design was based on a case-control study and included patients with NAFLD proven through biopsy and healthy controls.

\section{MATERIAL AND METHODS}

\section{Study design and selection of patients and controls}

Human serum samples from healthy donors and patients with NAFLD, and liver biopsies from all the patients were obtained with written informed consent under Institutional Review Board-approved protocols (protocol number 104/HGAZ/09 and 89/100).

This study was done in three phases: (i) global serum miRNA profiling with a human serum and plasma miRNA PCR-based array, which profiles the expression of 84 miRNAs detectable and differentially expressed in serum and plasma; miRNAs were selected by the manufacturer based on previous knowledge about published association of the expression of miRNAs in serum and plasma in the context of either liver or heart injury, CVD or liver disease, cancer and miRNAs routinely present in normal serum (a complete list of the miRNAs included in the array is provided in the online supplementary material and online supplementary table S1) (MIHS-106Z, Qiagen, Chatsworth, California USA) ( $\mathrm{n}=48$ participants). (ii) Independent validation of selected miRNAs based on their different patterns of expression and potential biological relevance to disease pathogenesis ( $\mathrm{n}=96$ participants). (iii) Evaluation of liver expression of candidate miRNAs ( $\mathrm{n}=65$ participants, 
51 NAFLD patients and the liver of 14 subjects without NAFLD (near-normal liver histology (NNLH).

Phases (i) and (ii) were conducted among independent groups of patients; phase (iii) involved liver tissue from patients recruited in phase (ii) and from patients with NNLH. Serum samples were collected by the time of liver biopsy in both cases and controls.

Patients were considered for inclusion at any study phase only if they had histopathological evidence of NAFLD, either SS or NASH, on liver biopsy done within the study period. Female and male patients with daily alcohol consumption of $>20$ and $30 \mathrm{~g}$, respectively, were not included in the study.

Health examinations included anthropometric measurements, a questionnaire on healthrelated behaviours, and biochemical determinations; measurements and blood sampling were obtained from each patient at the time of liver biopsy and before any intervention.

Specific biomarkers of inflammation (leucocyte count and $\mathrm{C}$ reactive protein) and atherogenesis (soluble intercellular adhesion molecule-1 (sICAM-1); plasminogen activator inhibitor-1 (PAI-1); soluble CD40 ligand; and resistin), and plasma caspase-generated cytokeratin-18 (CK-18) fragments were also measured.

Controls were selected from subjects whose age and sex matched those of the NAFLD patients. In addition to the standard health assessment described below, a careful ultrasonographic examination of the liver was done to exclude fatty liver infiltration. Controls were included at any study phase if they did not show features of MetSyn and did not have fatty liver at liver ultrasonography.

The reason for doing a liver biopsy in NNLH subjects was based on the presence of persistently mildly elevated serum liver enzyme activity. In all the NNLH subjects, all causes of common liver disease were ruled out, and these patients were included in the study if they did not present histological evidence of fatty change; the histological diagnosis was minimal changes or mild cholestasis.

\section{ISH for localisation and quantification of liver miR-122}

To localise and quantify candidate miRNAs in the liver, we performed in situ hybridisation (ISH) based on double digoxygenin-labelled locked nucleic acid oligos in formalin-fixed and paraffin-embedded tissue sections of NAFLD patients and controls.

\section{Cell culture and transfection of synthetic miR-122 mimic and miR-122 inhibitor}

To explore the effects of miR-122 on the regulation of liver enzymes, we used a LightSwitch MIM0136 hsa-miR-122-5p mimic, a chemically synthetic double-stranded RNA that acts as a functional equivalent of endogenous human miR-122, to transfect Huh-7 hepatoma cells.

Complete details regarding physical, anthropometric, biochemical, and cardiovascular evaluation, statistical analysis, liver biopsy and histopathological evaluation, miRNA isolation and quantification by real-time quantitative reverse-transcription PCR (RT-PCR) 
assay, immunoprecipitation of circulating miR-122 associated with argonaute2 (Ago2) complexes, liver immunohistochemistry (IHQ) and ISH, genotype analysis, pathway and target prediction, cell culture experiment, and a detailed summary study-flow chart are available in the online supplementary material.

\section{RESULTS}

Tables 1-3 show physical, anthropometric, biochemical and CVD evaluation, including biomarkers of atherosclerosis, of patients and controls in all the study phases.

\section{Circulating miRNAs}

\section{Differential circulating miRNA profile of NAFLD patients: results of serum miRNA PCR-based array (discovery analysis)—To map the circulating miRNA} expression profile in NAFLD, we first screened the serum of patients and controls by using a miRNA expression array. The heatmap diagram in figure 1 depicts a marked differential expression in several miRNAs in patients with SS and NASH when compared with controls; a heatmap of individual replicates is shown in the online supplementary material (supplementary figure S1). Among 84 miRNAs analysed, serum miR-122, miR-192, miR-19a and miR-19b, miR-125, and miR-375 were upregulated $>2$-fold $(\mathrm{p}<0.05)$ either in SS or NASH (figure 1). The most dramatic and significant fold changes were observed in the levels of miR-122 (7.2-fold change in NASH vs controls and 3.1-fold change in NASH vs SS) and miR-192 (4.4-fold change in NASH vs controls) (figure 1).

\section{Validation of selected circulating miRNAs by RT-PCR (confirmation analysis)}

Its role in the stratification of histological disease severity: We selected the six abovementioned significantly overexpressed miRNAs (see online supplementary table S2 for primers sequence) for validation in an independent and larger sample of well-characterised patients and controls. Regression analysis for an ordinal multinomial distribution showed that miR-122, miR-192 and miR-375 were significantly associated with the histological disease severity and significantly upregulated in NASH compared with SS patients ( $\mathrm{p}=0.006, \mathrm{p}=0.03$ and $\mathrm{p}=0.04$, respectively, in a non-parametric Mann-Whitney $\mathrm{U}$ test (figure 2). There were no gender differences in circulating miRNAs profiles and disease severity (ordinal multinomial probit, Wald statistic 0.13, p: 0.70).

We further explored the association between the expression level of these miRNAs and the individual components of the NAS in all the NAFLD patients $(n=77)$. Of note, by grouping the dependant variable according to NAS severity, hepatocellular ballooning, degree of liver fat infiltration, lobular inflammation and liver fibrosis, we observed a significant association between the serum levels of miR-122, miR-192 and miR-375 and both the NAS and cellular ballooning (figure 2). However, only the serum levels of miR-122 were significantly associated with the presence of advanced fibrosis by grouping fibrosis stage as $0-1$ versus 2-3 (figure 2).

Although the degree of steatosis was higher in the NASH group compared with SS patients, the serum levels of the explored miRNAs were not significantly associated with fatty liver infiltration (mild $₫ 3 \%$ vs moderate or severe $>33 \%$; see online supplementary figure 1 ). In 
addition, lobular inflammation was not associated with the circulating levels of the examined miRNAs (see online supplementary figure 1). Finally, a significant and strong correlation between miR-122 and liver enzymes was observed (table 4).

Receiver operating characteristic (ROC) curves were obtained to evaluate the performance of these miRNAs in predicting histological features associated with disease severity. The accuracies of miR-122, miR-192 and miR-375 to discriminate an advanced disease from a mild clinical form were fair and quite similar to each other (figure 2), showing on average an area under the ROC (AUROC) curve of about 0.7.

Furthermore, ROC analysis was adjusted by body mass index (BMI), and the results were similar to the unadjusted ones (see online supplementary material supplementary figure S2).

We also explored classic biomarkers used in the clinical setting to predict the presence of advanced liver disease, for example, a marker of cellular apoptosis (CK-18) and liver transaminases (alanine and aspartate aminotransaminases (ALT and AST). Figure 3 shows the overall performance of these biomarkers compared with miR-122 in predicting NASH and fibrosis in our population. Of note, miR-122 did much better than CK-18 and slightly better than ALT or AST in distinguishing patients with NASH and in predicting liver fibrosis (figure 3). Interestingly, and not totally unexpected, we observed that miR-122 and miR-192 were significantly and positively correlated with serum CK-18 levels $(\mathrm{R}=0.4$, $\mathrm{p}<0.03$ and $\mathrm{R}=0.37, \mathrm{p}<0.03$, respectively).

Finally, we performed multivariate ROC analysis and observed that the incorporation of multiple biomarkers into the model did not perform better to predict the disease severity, and miR-122 is as good as transaminases in discriminating a NAS score above 5 (see online supplementary material, online supplementary figures S3-S4 and online supplementary table S5).

\section{Circulating miRNAs and its relationship with features of the MetSyn: Next, we} attempted to confirm the differential expression observed for miR-19a and miR-19b and for miR-125b, which was significantly associated with NAFLD in the screening phase; miR-19a and mir-19b belong to the same cluster of miRNAs located in Chr13 (see online supplementary figure 2). In the replication stage, these miRNAs were significantly higher in SS and NASH compared with controls, but they did not differ between SS and NASH (see online supplementary figure 2). Nevertheless, these miRNAs were significantly associated with features of the MetSyn (fasting plasma glucose, triglyceride levels and BMI) and biomarkers of CVD risk and atherogenesis (sICAM-1 and PAI-1 levels) (table 4). miR19a/b and miR-125b were also involved in disease pathways associated with metabolic disorders, regulation of blood pressure and blood coagulation, including formation of thrombin (see online supplementary material and online supplementary table S6).

\section{Role of Ago2-ribonucleoprotein complexes in the release of miR-122 into the circulation in patients with NAFLD-Argonaute proteins are the direct binding partners of small RNAs, and human Ago2 is associated with the catalytic activity required for mRNA cleavage. ${ }^{10}$ Of note, a previous study found that miRNAs, including miR-122,}


circulate in plasma/serum in vesicle-free ribonucleoprotein complexes, in association with Ago 2. ${ }^{11}$ Moreover, it was proposed that miR-122 is released through a protein carrier pathway because it was only detected in protein-associated fractions. ${ }^{12}$

Thus, to explore whether circulating miR-122 in NAFLD patients is primarily exported in association with Ago2 protein, we quantified its expression in Ago2-linked and Ago2-free fractions in circulation.

We showed that the relative abundance of circulating miR-122 in NAFLD patients was higher in the Ago2-free supernatant compared with the amount that immunoprecipi-tated with the anti-Ago2 antibody; patients with NASH and SS showed a significant enrichment (>10-fold and sevenfold, respectively) of free miR-122 in the circulation compared with that in association with Ago2 complexes (figure 4). Nevertheless, a small fraction (around 25\%) of miR-122 circulates in serum in association with Ago2; this maintains the same pattern of higher levels in NASH observed in the Ago2-free pool. Of note, the Ago2 complexes are several-fold enriched in miR-122 with respect to miR-23a; after normalisation, the ratio of miR-122 in the immunoprecipitate/supernatant is completely reversed (data not shown).

To explore the source of circulating miR-122-Ago2 complexes, we further evaluated by IHQ the expression of Ago2 protein in liver tissue of NAFLD patients with different degrees of histological severity and in the liver of NNLH subjects. Ago2 was barely expressed in controls; the immunoreactivity product of Ago2 in NAFLD was confined to the cytoplasm of hepatocytes, primarily but not exclusively restricted to areas loaded with lipids (figure 4). In addition, Ago2 was expressed at the edge of the wall of hepatocytes. The staining scores according to the disease status showed that the liver expression of Ago2 had the same pattern as that of miR-122 observed by ISH, which was significantly higher in patients with SS compared with NASH patients (figure 4). In summary, we may suggest that miR-122 and Ago2 are colocalised in the hepatocyte and that serum miR-122 in Ago2-free and Ago2 complexes may have the same origin.

\section{Genetic variation of a validated miR-122 target gene: a candidate-gene association study miR-122-related sequence genetic variation in the $3^{\prime} U T R$ of the L-arginine transporter gene (SLC7A1) is associated with arterial hypertension-A}

functional variant within the $3^{\prime}$-untranslated region (UTR) of the SLC7Al gene (rs41318021 $\mathrm{C} / \mathrm{T}$, formerly submitted as ss52051826) was previously associated with essential hypertension. ${ }^{13}$ The results of experimental models showed that changes in the expression of $S L C 7 A 1$ were associated with changes in nitric oxide production leading to endothelial dysfunction. ${ }^{13}$ The rs 41318021 variant is associated with a miR-122 binding site that is involved in controlling SLC7Al gene expression. ${ }^{14}$

Hence, to explore the hypothesis of a putative relationship among miR-122, the rs 41318021 variant and features of the MetSyn, we included 248 individuals (100 subjects without fatty liver and 148 NAFLD patients) in the analysis; details are given in the online supplementary material. Case-control association analysis of rs4131802 was done through logistic analysis. The genotype distribution was in Hardy-Weinberg equilibrium. 
Interestingly, we found that the rs41318021 variant was significantly associated with arterial systolic/diastolic hypertension (Pearson $\chi^{2}: 7.85, \mathrm{p}<0.019$ ); the distribution of genotypes according to disease trait is provided in the online supplementary material (supplementary table S4). In the dominant model, the OR for arterial hypertension (OR, 5.01; 95\% CI 1.79 to $14.00 ; \mathrm{p}<0.003$ ) or isolated diastolic hypertension (OR, 4.54; 95\% CI 1.49 to 13.87 ; $\mathrm{p}<0.008$ ) was still significant even after adjusting for age, BMI and fatty liver. These findings suggest that the $3^{\prime}$ UTR variant in human SLC7A1 may increase susceptibility to arterial hypertension in patients with NAFLD by interacting with miR-122, which is significantly upregulated in circulation, as previously shown. Remarkably, the expression levels of miR-122 in liver, which feed the higher levels in circulation, significantly and negatively correlate with systolic arterial blood pressure $(R=-0.46, p<0.01)$.

\section{Liver expression of candidate miRNAs}

\section{Expression pattern of liver miR-122 in NAFLD patients according to disease} severity and tissue localisation-To explore the pattern of liver miR-122 expression and its relationship with the histological disease severity, we quantified the miR-122 expression levels in NAFLD compared with NNLH patients. Surprisingly, NASH patients showed a systematic downregulation in the liver expression of those miRNAs that were upregulated in serum, for instance, miR-122 and miR-192 (figure 5). Of note, the liver expression of miR-122 and miR-192 was 10-fold ( $\mathrm{p}<0.03)$ and almost twofold ( $<<0.03)$ downregulated, respectively, in NASH patients compared with SS (figure 5); the liver expressions of miR-122 and miR-192 were significantly correlated $(\mathrm{R}=0.56, \mathrm{p}=0.00004)$.

In addition, we used ISH to study the distribution of liver miR-122 and putatively understand its release into the circulation and to validate the results of qPCR expression. We con-firmed that miR-122 is preferentially localised at the edge of lipid-laden hepatocytes being homogeneously distributed throughout the lobule without zone preferences but with high accentuation in areas of fat degeneration and zone 3.

In addition, we noticed that higher levels of miR-122 were expressed at the edge of the wall of hepatocytes rather than in the cytoplasm, suggesting that miR-122 is ready to be exported into the circulation (figure 5). Furthermore, we observed that NASH patients had significantly lower miR-122-hybridisation marks than did patients with SS (figure 5).

Finally, liver miR-122 expression was significantly and inversely correlated with central obesity $(\mathrm{R}=-0.40, \mathrm{p}=0.03)$, serum low-density lipoprotein-cholesterol $(\mathrm{R}=-0.30, \mathrm{p}=0.04)$ and serum alkaline phosphatase levels $(\mathrm{R}=-0.42, \mathrm{p}=0.003)$.

\section{In vitro studies to examine putative mechanistic pathways of the relationship between miR-122 and transaminases}

miR-122 positively regulates alanine aminotransferase activity-The dysregulation of miR-122 expression is known to affect normal liver function and metabolism; ${ }^{15}$ thus, we hypothesised that miR-122 and liver transaminases (ALT or GPT and AST or GOT), which have as a common source the liver and which levels were significantly higher in the circulation in NAFLD, might share underlying regulatory 
mechanisms. We performed an in vitro study in which we both overexpressed and blocked the endogenous miR-122 activity (details are provided in the online supplementary material) to explore putative changes in the liver transcriptional activity of aminotransferases genes (see online supplementary table S3 for primers sequence); toward this end, we transfected Huh7 cells with synthetic miR-122 mimic and its inhibitor.

We observed that the overexpression of miR-122 significantly enhanced ALT but not AST enzymatic activity in liver cell lysates (Kruskal-Wallis Test: $\mathrm{p}<0.02$; figure 6). Interestingly, the GPT1 (the gene that encodes the cytosolic alanine aminotransaminase 1 (ALT1), GOT1 (the gene that encodes the cytoplasmic form of glutamic-oxaloacetic transaminase, AST) and GOT2 (the gene that encodes the mitochondrial form of glutamic-oxaloacetic transaminase) mRNA levels did not change during either miR-122 overexpression or lossof-function experiments (figure 6). This novel finding suggests that miR-122 upregulates the translation of the ALT (GPT1) protein, leading to increased enzymatic activity. Although it is common knowledge that miRNAs usually repress gene transcription by degrading target mRNAs, induction of protein translation has been previously reported. ${ }^{16}$ Surprisingly, Ago2, which is highly expressed in NAFLD compared with control liver, is involved in the mechanisms that lead to translation activation by interacting with miRNA-responsive elements. ${ }^{16}$

In order to replicate our in vitro findings, we evaluated the expression of GPTI mRNA in the liver of NAFLD patients, and similar to the observations in Huh7 cells, the expression levels of miR-122 and GPT1 mRNA were not significantly associated, suggesting that miR-122 does not influence liver GPTI transcription.

\section{Target prediction: miR-122 has predicted complementary sites located in the coding region of GPT1 mRNA-miRNAs usually repress gene expression by} interfering with their target-mRNA gene by base pairing in the untranslated regions ( $3^{\prime}$-UTR and 5'-UTR) inducing mRNA destabilisation and translational inhibition; binding to the $5^{\prime}$ UTR can either repress or activate translation. Consistent with our experimental results, in silico sequence analysis by five well-established programmes for miRNA and 3'-UTR-target interactions (TargetScan, PITA, miRanda, miRBase and PicTar) showed that GPT1 3'-UTR region has not predicted miR-122-target sites. Likewise, we used specific bioinformatics approaches for miRNA-target sites in 5'-UTRs (miRTar 2, Sfold-STarMirDB, MiRNA_Targets 2012 and Mircode) and observed that miR-122 does not target the GPT1 5 '-UTR region.

Previous observations demonstrated that coding sequence-located miRNA target sites are able to stimulate protein expression. ${ }^{17}$ Given that miR-122 upregulated in vitro the activity of GPT protein, we speculated that the binding interaction between miR-122 and GPTI might not reside in the UTR regions. Thus, we explored for putative miR-122 target sites on the whole $G P T 1$ cDNA sequence by free energy-based miRNA prediction programmes (PITA and MicroInspector), and found six putative miR-122-target sites, including one located in position 137 with a high energy score, suggesting that miR-122 could possibly interact with GPTI at multiple sites of the coding region (see online supplementary material and online supplementary table S7). 


\section{In silico analysis of pathways associated with dysregulated miRNAs in NAFLD}

Rather than exploring the putative hundreds of target genes associated with dysregulated miRNAs in NAFLD, we mapped the disease pathways in which these genes are involved (see online supplementary figure 3). The pathways associated with the MetSyn in which the miRNA-associated target genes are involved are provided in the online supplementary table S6. Of note, miR-19a/b and miR-125b are involved in biological processes that support their association with an altered CVD risk profile. In addition, the targets of the dysregulated miRNAs belong to a highly interacting network; interestingly, some targets were previously reported to have altered mRNA in NASH versus SS patients, ${ }^{18}$ including but not limited to, transforming growth factor- $\beta 1$ (TGF- $\beta 1$ ) (see online supplementary table S6).

\section{DISCUSSION}

This multiphase case-control study explored the role of circulating miRNAs in NAFLD patients at different stages of histological disease severity; we performed an initial step of global exploration of circulating miRNAs, followed by two steps of individual RTPCR evaluation, one in serum and another in liver tissue. In the training sera samples, we identified six miRNAs, including miR-122, miR-192, miR-375, miR-19a and miR-19b, and miR-125b, the concentrations of which were significantly upregulated in patients with NAFLD compared with healthy controls. ROC analysis in the validation samples revealed that three miRNAs, including miR-122, miR-192 and miR-375, have the potential to distinguish NASH from SS, but only miR-122 distinguishes liver fibrosis. We also compared the predictive value of serum miR-122 and classic disease biomarkers, including CK-18, ALT and AST, and we observed that miR-122 was slightly superior in predicting $\mathrm{NASH}$ and fibrosis in our population (AUROC 0.714). Although this predictive value is not good enough for an ideal biomarker, the performance of miR-122 is quite similar to that of CK-18 in predicting NASH (AUROC 0.65) and fibrosis (AUROC 0.68), as recently reported in a multicentre study. ${ }^{19}$ Consequently, there is no accurate non-invasive disease biomarker for distinguishing NASH from SS, and bio-marker discovery still remains a big challenge.

Interestingly, miR-122 and miR-192 significantly correlated with CK-18 levels, suggesting that they mirror a common pathophysiological condition, such as a caspase-related apoptosis pathway.

A remarkable finding to highlight is the putative role of circulating miR-122 as an extrahepatic fingerprint of NASH. For instance, in our study, the liver expression of miR-122 was significantly decreased in NASH, as previously shown by Cheung et al. ${ }^{20}$ In addition, the localisation of hybridisation marks showed a consistent pattern of miR-122 expression at the edge of the wall of hepatocytes, suggesting that miR-122 is ready to be exported into the circulation. Overall, these findings suggest that the lower expression of miR-122 in liver is a consequence of a high rate of release into the circulation rather than a down-regulation of miRNA expression per se. In fact, the inverse relationship between circulating and tissue expression of miR-122 seems to be the consequence of a dynamic regulation of the biology of miR-122 in the liver. This phenomenon generates a dynamics of miR-122 production/release that reflects the behaviour of flux of substances/molecules between compartments. Accordingly, there are multiple reports, including a wide range of 
diseases associated with liver injury (from acute viral hepatitis and drug-induced liver injury to liver cancer), that validated the opposite expression pattern of circulating and liver miR-122. ${ }^{21-23}$ Thus, collectively, these results indicate that the inverse relationship between tissue and circulating miR-122 may reflect a phenomenon of physiological significance in the biology of liver diseases, including NAFLD. Finally, it is hard to infer mechanisms from the analysis of the steady state levels of interconnected compartments, but as the final and largest reservoir may be the circulation, it is tempting to speculate that the overall production of miR-122 is increased in NAFLD.

In addition, serum miR-122 was significantly associated with hepatocellular ballooning, suggesting a putative role of this miRNA in the pathogenesis of NAFLD because this histological feature represents a morphological hallmark of NASH involving degenerative changes, a dilated endoplasmic reticulum and cytoskeletal injury. ${ }^{24}$ Hence, circulating miR-122 might be an interesting biomarker in monitoring the progression of histological changes, for instance, during therapeutic intervention. Furthermore, serum miR-122 levels were significantly associated with fibrosis scores, but liver miR-122 expression was not associated with either histological grading or staging. Notably, a recent in vitro study showed that miR-122 expression was downregulated in activated primary rat hepatic stellate cells (HSCs) suggesting a role of miR-122 in the regulation of collagen production. ${ }^{25}$ Therefore, it might be interesting to explore in further studies the behaviour of miRNAs in HSCs from NASH patients to confirm whether these cells are able to express miR-122.

Circulating miR-122 has also been proposed as an early disease severity-dependent biomarker of liver injury (viral, alcohol and drug-related) because its levels become elevated earlier than that of serum ALT. ${ }^{2627}$ We observed that in NAFLD patients, miR-122 is strongly correlated with serum ALT; thus, to better understand this relationship, we explored in vitro the gene expression levels and the enzymatic activity of GPT1, GOT1 and GOT2, and ALT and AST, respectively, in Huh-7 hepatoma cells that either overexpress or have blocked miR-122. We uncovered a novel finding in that miR-122 played a regulatory role in the levels of ALT without changing the transcriptional levels of the coding gene.

Surprisingly, over-expression of miR-122 caused a significant increase in ALT enzymatic activity compared with cells transfected with a scramble miRNA or a miR-122 inhibitor, suggesting that this miRNA is involved in translation activation of ALT. Our findings are supported by previous studies showing that miRNA-protein complexes oscillate between repression and activation; for instance, Vasudevan et al $^{16}$ identified a miRNA (miR-369-3) that, in direct association with Ago2, is able to activate translation of its target gene. Similarly, Henke et $\mathrm{l}^{28}$ showed that miR-122 enhances the efficiency of HCV protein translation. In addition, target site prediction analysis using full-length cDNA sequence of GPTl revealed at least six potential miR-122 target sites, which may interact cooperatively to enhance protein translation. Similar results of translation upre-gulation without altering mRNA levels were previously reported; ${ }^{29}$ moreover, the ENCODE Project demonstrated that miRNAs are able to bind multiple non-UTR sites in their target genes. ${ }^{30}$ Furthermore, although more experiments should be done, recent evidence showed that substantial fraction of human miRNA target sites are non-canonical. ${ }^{31}$ 
The fact that ALT is involved in cellular nitrogen metabolism and in liver gluconeogenesis by catalysing the transamination between alanine and 2-oxoglutarate suggests a novel role of miR-122 in the modulation of glucose metabolism that has not been previously reported. Interestingly, the preferential localisation of miR-122 around lipid droplets of the hepatocytes reinforces this concept.

Contrary to the findings of Arroyo et al, ${ }^{11}$ we observed that extracellular Ago2-miR-122 complexes represent a small fraction of the serum of NAFLD patients. As shown in experimental studies, miR-122 mostly circulates in Ago2-free complexes. ${ }^{26}$ Nevertheless, Ago2, a member of the argonaute protein family involved in the RNA-induced silencing complex, was found to play a role in the pathogenesis of NAFLD because its liver expression showed a significant upregulation that followed the same expression pattern as that of liver miR-122. Unfortunately, the limitations of our technique prevented us from proving colocalisation. However, we showed that circulating miR-122, either in other forms or in Ago2 complexes, seems to originate from the same cell type and reflects the same underlying disorder.

Another interesting finding of our study is the significant association of miR-192 and miR-375 with the disease severity. Indeed, the dysregulation of miR-192, which is upregulated by $T G F \beta 1,{ }^{32}$ and miR-375, which is a key regulator of glucose homeostasis and essential for adaptive $\beta$-cell expansion in response to increasing insulin demand in insulin resistance, ${ }^{33}$ confirms the importance of these miRNAs in the pathogenesis of NAFLD. In agreement with these findings, we previously observed in our population that the liver $T G F \beta 1$ expression was sevenfold increased in NASH compared with SS. ${ }^{18}$ Hence, miR-192 is clearly a profibrogenic molecule involved in fibrosis development in many other tissues such as in kidney by activation of TGF- $\beta /$ Smad signalling. ${ }^{34}$ Moreover, we hypothesise that the increased circulating miRNA levels may have some impact on the MetSyn phenotypes. Because miR-122 showed higher levels in circulation, we first looked at a functional polymorphism in the $3^{\prime}$ UTR region of a validated miR-122 target gene (SLC7A1), the expression of which is affected by miRNA. We observed that rs41318021 was significantly associated with arterial hypertension, suggesting that miR-122 might potentially modulate the susceptibility to elevated blood pressure in subjects who carry the risk allele of this variant. Our results might suggest a scenario in which circulating levels of miR-122 might regulate the expression of genes involved in endothelial damage, for example, SLC7A1. ${ }^{514}$

Besides, among the dysregulated miRNAs in circulation, we identified a set of miRNAs that were unique to only the risk of CVD and atherogenesis in patients with NAFLD, such as $\mathrm{miR}-19 \mathrm{a} / \mathrm{b}$ (associated with NF- $\mathrm{kB}$ signalling) ${ }^{35}$ and miR-125b (associated with inflammation). ${ }^{36}$ Interestingly, these miRNAs were upregulated in NAFLD patients without any relation to the disease severity, suggesting that the underlying systemic inflammatory state associated with fatty liver is irrespective of the histological status. Accordingly, in silico analysis of pathways under the miRNA-network regulation revealed that miR19b/a and miR-125b are critically involved in metabolic homeostasis, complement and coagulation cascades, glutathione metabolism, and the reninangiotensin system. 
Given that about $60 \%$ of all mammalian protein-encoding genes are regulated at posttranscriptional level by miRNAs, ${ }^{37}$ any explanation by which the dysregulated miRNAs in our study modulate MetSyn might result in insufficiency. Nevertheless, accumulating evidence supports the metabolic and molecular mechanisms whereby miRNAs might potentially explain the association between fatty liver and MetSyn-associated diseases; ${ }^{38}$ for instance, the inflammation-associated miR-125b that is involved in vascular dysfunction and associated with increased oxidant stress and insulin resistance in diabetic mice, ${ }^{36}$ or the liver specific miR-122, which not only has a significant impact on lipid homeostasis ${ }^{3940}$ but is involved in cardiac fibrosis by modulating myocardial $T G F \beta 1$ expression. ${ }^{41}$ Furthermore, dysregulated cholesterol metabolism in NAFLD, which may contribute to disease severity and cardiovascular risks, is regulated by miRNAs. ${ }^{42}$

In conclusion, our results suggest that NAFLD has a distinct circulating miRNA signature, which is a reliable fingerprint of the morphological changes occurring in liver tissue. In addition, by characterising the dysregulated miRNAs in the circulation, we might be able to identify key signalling pathways involved in the pathogenesis of the disease and its relationship with the MetSyn; hence, miRNA profiling could also identify individuals at risk.

\section{Supplementary Material}

Refer to Web version on PubMed Central for supplementary material.

\section{Acknowledgments}

Funding This study was partially supported by grants PICT 2010-0441 and PICT 2012-0159 (Agencia Nacional de Promoción Científica y Tecnológica), UBACYT CM04 (Universidad de Buenos Aires), and CONICET (Res. 1038 to Dr. Sookoian, Scientific Exchange in the context of International Cooperation Agreements NSF-CONICET).

\section{References}

1. Selbach M, Schwanhausser B, Thierfelder N, et al. Widespread changes in protein synthesis induced by microRNAs. Nature. 2008; 455:58-63. [PubMed: 18668040]

2. Weiland M, Gao XH, Zhou L, et al. Small RNAs have a large impact: circulating microRNAs as biomarkers for human diseases. RNA Biol. 2012; 9:850-9. [PubMed: 22699556]

3. Creemers EE, Tijsen AJ, Pinto YM. Circulating microRNAs: novel biomarkers and extracellular communicators in cardiovascular disease? Circ Res. 2012; 110:483-95. [PubMed: 22302755]

4. Redis RS, Calin S, Yang Y, et al. Cell-to-cell miRNA transfer: from body homeostasis to therapy. Pharmacol Ther. 2012; 136:169-74. [PubMed: 22903157]

5. Pirola CJ, Gianotti TF, Castano GO, et al. Circulating MicroRNA-122 signature in nonalcoholic fatty liver disease and cardiovascular disease: a new endocrine system in metabolic syndrome. Hepatology. 2013; 57:2545-7. [PubMed: 23111985]

6. Brunt EM, Kleiner DE, Wilson LA, et al. Nonalcoholic fatty liver disease (NAFLD) activity score and the histopathologic diagnosis in NAFLD: distinct clinicopathologic meanings. Hepatology. 2011; 53:810-20. [PubMed: 21319198]

7. Sookoian S, Rosselli MS, Gemma C, et al. Epigenetic regulation of insulin resistance in nonalcoholic fatty liver disease: impact of liver methylation of the peroxisome proliferator-activated receptor gamma coactivator 1alpha promoter. Hepatology. 2010; 52:1992-2000. [PubMed: 20890895] 
8. Pirola CJ, Gianotti TF, Burgueno AL, et al. Epigenetic modification of liver mitochondrial DNA is associated with histological severity of nonalcoholic fatty liver disease. Gut. 2013; 62:1356-63. [PubMed: 22879518]

9. Lonardo A, Sookoian S, Chonchol M, et al. Cardiovascular and systemic risk in nonalcoholic fatty liver disease-atherosclerosis as a major player in the natural course of NAFLD. Curr Pharm Des. 2013; 19:5177-92. [PubMed: 23432668]

10. Meister G, Landthaler M, Patkaniowska A, et al. Human Argonaute2 mediates RNA cleavage targeted by miRNAs and siRNAs. Mol Cell. 2004; 15:185-97. [PubMed: 15260970]

11. Arroyo JD, Chevillet JR, Kroh EM, et al. Argonaute2 complexes carry a population of circulating microRNAs independent of vesicles in human plasma. Proc Natl Acad Sci U S A. 2011; 108:5003-8. [PubMed: 21383194]

12. Chang J, Nicolas E, Marks D, et al. miR-122, a mammalian liver-specific microRNA, is processed from hor mRNA and may downregulate the high affinity cationic amino acid transporter CAT-1. RNA Biol. 2004; 1:106-13. [PubMed: 17179747]

13. Yang Z, Venardos K, Jones E, et al. Identification of a novel polymorphism in the $3^{\prime}$ UTR of the Larginine transporter gene SLC7A1: contribution to hypertension and endothelial dysfunction. Circulation. 2007; 115:1269-74. [PubMed: 17325243]

14. Yang Z, Kaye DM. Mechanistic insights into the link between a polymorphism of the $3^{\prime}$ UTR of the SLC7A1 gene and hypertension. Hum Mutat. 2009; 30:328-33. [PubMed: 19067360]

15. Krutzfeldt J, Rajewsky N, Braich R, et al. Silencing of microRNAs in vivo with 'antagomirs'. Nature. 2005; 438:685-9. [PubMed: 16258535]

16. Vasudevan S, Tong Y, Steitz JA. Switching from repression to activation: microRNAs can upregulate translation. Science. 2007; 318:1931-4. [PubMed: 18048652]

17. Tay Y, Zhang J, Thomson AM, et al. MicroRNAs to Nanog, Oct4 and Sox 2 coding regions modulate embryonic stem cell differentiation. Nature. 2008; 455:1124-8. [PubMed: 18806776]

18. Sookoian S, Gianotti TF, Rosselli MS, et al. Liver transcriptional profile of atherosclerosis-related genes in human nonalcoholic fatty liver disease. Atherosclerosis. 2011; 218:378-85. [PubMed: 21664615]

19. Cusi K, Chang Z, Harrison S, et al. Limited value of plasma cytokeratin-18 as a biomarker for NASH and fibrosis in patients with non-alcoholic fatty liver disease. J Hepatol. 2014; 60:167-74. [PubMed: 23973932]

20. Cheung O, Puri P, Eicken C, et al. Nonalcoholic steatohepatitis is associated with altered hepatic MicroRNA expression. Hepatology. 2008; 48:1810-20. [PubMed: 19030170]

21. Choi Y, Dienes HP, Krawczynski K. Kinetics of miR-122 expression in the liver during acute HCV infection. PLoS ONE. 2013; 8:e76501. [PubMed: 24124569]

22. Coulouarn C, Factor VM, Andersen JB, et al. Loss of miR-122 expression in liver cancer correlates with suppression of the hepatic phenotype and gain of metastatic properties. Oncogene. 2009; 28:3526-36. [PubMed: 19617899]

23. Wang K, Zhang S, Marzolf B, et al. Circulating microRNAs, potential biomarkers for druginduced liver injury. Proc Natl Acad Sci U S A. 2009; 106:4402-7. [PubMed: 19246379]

24. Caldwell S, Ikura Y, Dias D, et al. Hepatocellular ballooning in NASH. J Hepatol. 2010; 53:71923. [PubMed: 20624660]

25. Li J, Ghazwani M, Zhang Y, et al. miR-122 regulates collagen production via targeting hepatic stellate cells and suppressing P4HA1 expression. J Hepatol. 2013; 58:522-8. [PubMed: 23178710]

26. Bala S, Petrasek J, Mundkur S, et al. Circulating microRNAs in exosomes indicate hepatocyte injury and inflammation in alcoholic, drug-induced and inflammatory liver diseases. Hepatology. 2012; 56:1946-57. [PubMed: 22684891]

27. Zhang Y, Jia Y, Zheng R, et al. Plasma microRNA-122 as a biomarker for viral-, alcohol-, and chemical-related hepatic diseases. Clin Chem. 2010; 56:1830-8. [PubMed: 20930130]

28. Henke JI, Goergen D, Zheng J, et al. microRNA-122 stimulates translation of hepatitis C virus RNA. EMBO J. 2008; 27:3300-10. [PubMed: 19020517]

29. Lu H, Buchan RJ, Cook SA. MicroRNA-223 regulates Glut4 expression and cardiomyocyte glucose metabolism. Cardiovasc Res. 2010; 86:410-20. [PubMed: 20080987] 
30. Birney E, Stamatoyannopoulos JA, Dutta A, et al. Identification and analysis of functional elements in $1 \%$ of the human genome by the ENCODE pilot project. Nature. 2007; 447:799-816. [PubMed: 17571346]

31. Khorshid M, Hausser J, Zavolan M, et al. A biophysical miRNA-mRNA interaction model infers canonical and noncanonical targets. Nat Methods. 2013; 10:253-5. [PubMed: 23334102]

32. Kato M, Zhang J, Wang M, et al. MicroRNA-192 in diabetic kidney glomeruli and its function in TGF-beta-induced collagen expression via inhibition of E-box repressors. Proc Natl Acad Sci U S A. 2007; 104:3432-7. [PubMed: 17360662]

33. Poy MN, Hausser J, Trajkovski M, et al. miR-375 maintains normal pancreatic alpha- and beta-cell mass. Proc Natl Acad Sci U S A. 2009; 106:5813-18. [PubMed: 19289822]

34. Chung AC, Huang XR, Meng X, et al. miR-192 mediates TGF-beta/Smad3-driven renal fibrosis. J Am Soc Nephrol. 2010; 21:1317-25. [PubMed: 20488955]

35. Gantier MP, Stunden HJ, McCoy CE, et al. A miR-19 regulon that controls NF-kappaB signaling. Nucleic Acids Res. 2012; 40:8048-58. [PubMed: 22684508]

36. Villeneuve LM, Kato M, Reddy MA, et al. Enhanced levels of microRNA-125b in vascular smooth muscle cells of diabetic $\mathrm{db} / \mathrm{db}$ mice lead to increased inflammatory gene expression by targeting the histone methyltransferase Suv39h1. Diabetes. 2010; 59:2904-15. [PubMed: 20699419]

37. Friedman RC, Farh KK, Burge CB, et al. Most mammalian mRNAs are conserved targets of microRNAs. Genome Res. 2009; 19:92-105. [PubMed: 18955434]

38. Zampetaki A, Mayr M. MicroRNAs in vascular and metabolic disease. Circ Res. 2012; 110:50822. [PubMed: 22302757]

39. Elmen J, Lindow M, Silahtaroglu A, et al. Antagonism of microRNA-122 in mice by systemically administered LNA-antimiR leads to up-regulation of a large set of predicted target mRNAs in the liver. Nucleic Acids Res. 2008; 36:1153-62. [PubMed: 18158304]

40. Tsai WC, Hsu SD, Hsu CS, et al. MicroRNA-122 plays a critical role in liver homeostasis and hepatocarcinogenesis. J Clin Invest. 2012; 122:2884-97. [PubMed: 22820290]

41. Beaumont J, Lopez B, Hermida N, et al. microRNA-122 down-regulation may play a role in severe myocardial fibrosis in human aortic stenosis through TGF-beta1 up-regulation. Clin Sci (Lond). 2014; 126:497-506. [PubMed: 24168656]

42. Min HK, Kapoor A, Fuchs M, et al. Increased hepatic synthesis and dysregulation of cholesterol metabolism is associated with the severity of nonalcoholic fatty liver disease. Cell Metab. 2012; 15:665-74. [PubMed: 22560219] 


\section{Significance of this study}

\section{What is already known on this subject?}

- MicroRNA (miRNAs) are small RNA molecules that participate in the posttranscriptional fine tuning of gene regulation.

- Circulating miRNAs have been proposed as attractive diagnostic tools for distinguishing, non-invasively, diseased individuals from healthy ones.

\section{What are the new findings?}

- miR-122 (which constitutes more than 70\% of the total liver miRNA pool), miR-192 (upregulated by TGF $\beta 1$ ) and miR-375 (a key regulator of glucose homeostasis) were significantly elevated in non-alcoholic steatohepatitis (NASH) compared with simple steatosis. NASH patients showed a systematic downregulation in the liver expression of those miRNAs that were upregulated in serum.

- We uncovered novel findings about miR-122 and the biology of non-alcoholic fatty liver disease (NAFLD): miR-122 mostly circulates in Ago2-free complexes and is expressed by the lipid-laden hepatocytes. In Huh7 cells, miR-122 regulates the levels of ALT without changing the transcriptional level of the coding gene, suggesting that it is involved in translation activation of ALT; miR-122 might interact with GPT1 (ALT) at multiple sites of the coding region to enhance translation. A functional variant (rs41318021) in the $3^{\prime} \mathrm{UTR}$ region of a validated miR-122 target gene involved in endothelial damage (SLC7A1) was significantly associated with arterial hypertension. The expression levels of liver miR-122 significantly and negatively correlated with systolic arterial blood pressure.

- NAFLD has a distinguishing circulating miRNA profile associated with a global dysmetabolic disease state and cardiovascular disease risk, such as miR-19a/b (associated with NF- $\mathrm{kB}$ signalling) and miR-125b (associated with inflammation).

\section{How might it impact on clinical practice in the foreseeable future?}

- The circulating miRNA signature of NAFLD mirrors the molecular events occurring in the liver and provides clues for the understanding of the disease biology.

- The application in the clinical setting of circulating miRNAs as non-invasive disease biomarkers is still challenging as its performance is similar to that of ALT or CK-18 for predicting NASH.

- miRNAs might potentially explain the association between fatty liver and cardiovascular disease. 


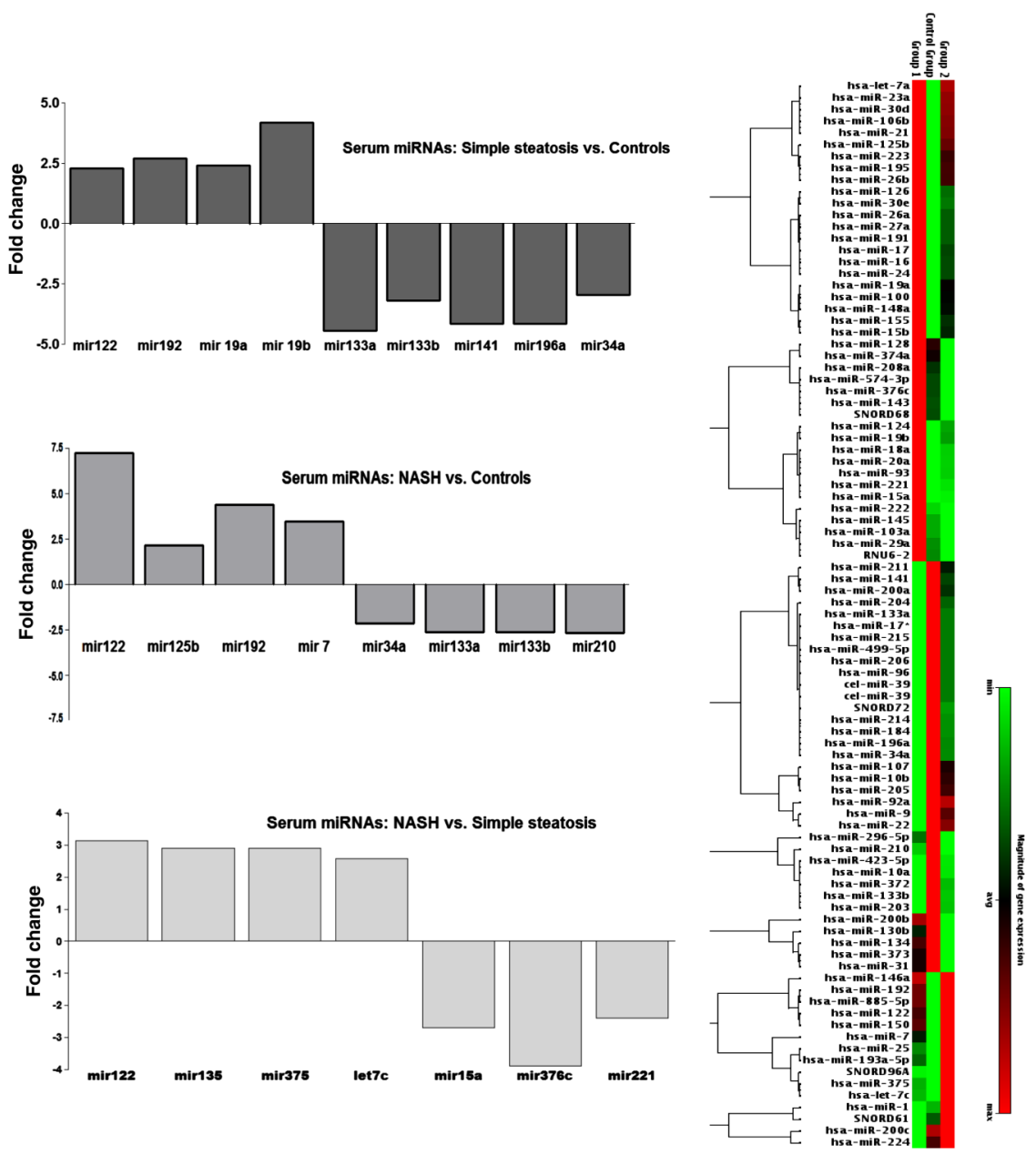

Figure 1.

Global circulating miRNA expression profile in non-alcoholic fatty liver disease (NAFLD) patients and control subjects. Left, a heat map shows the results of 84 differentially expressed miRNAs in NAFLD patients compared with healthy controls. Red, overexpressed miRNAs; green, downregulated miRNAs; black, no change. The three groups of samples: control group, group 1 (simple steatosis) and group 2 (non-alcoholic steatohepatitis), can be distinguished according to the profile. Right, bars show the fold changes of significantly dysregulated miRNAs $(\mathrm{p}<0.05)$ according to disease severity. 

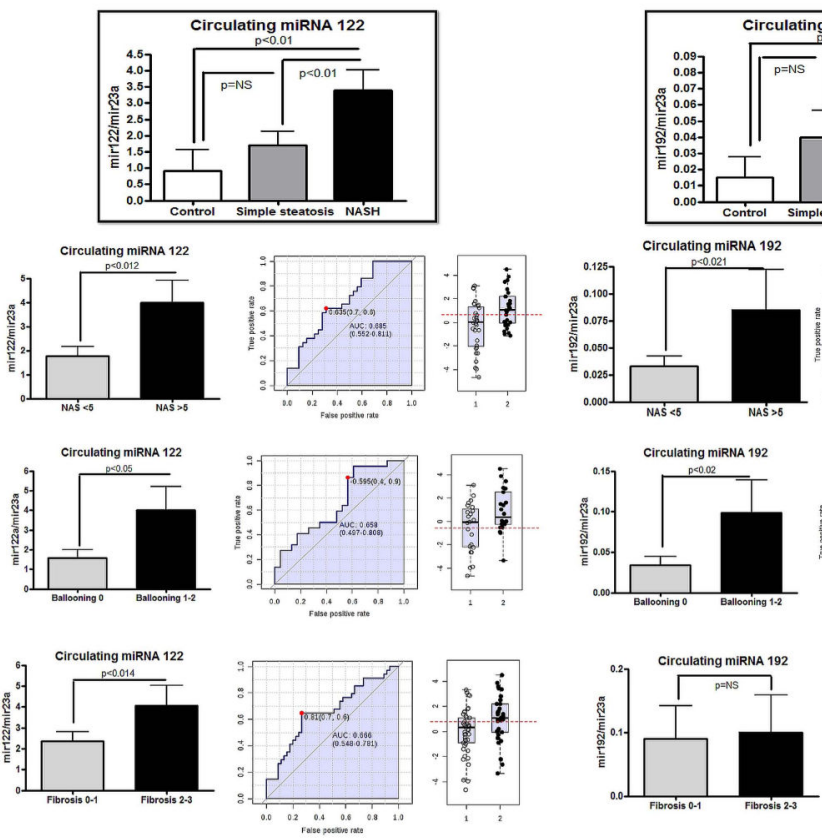
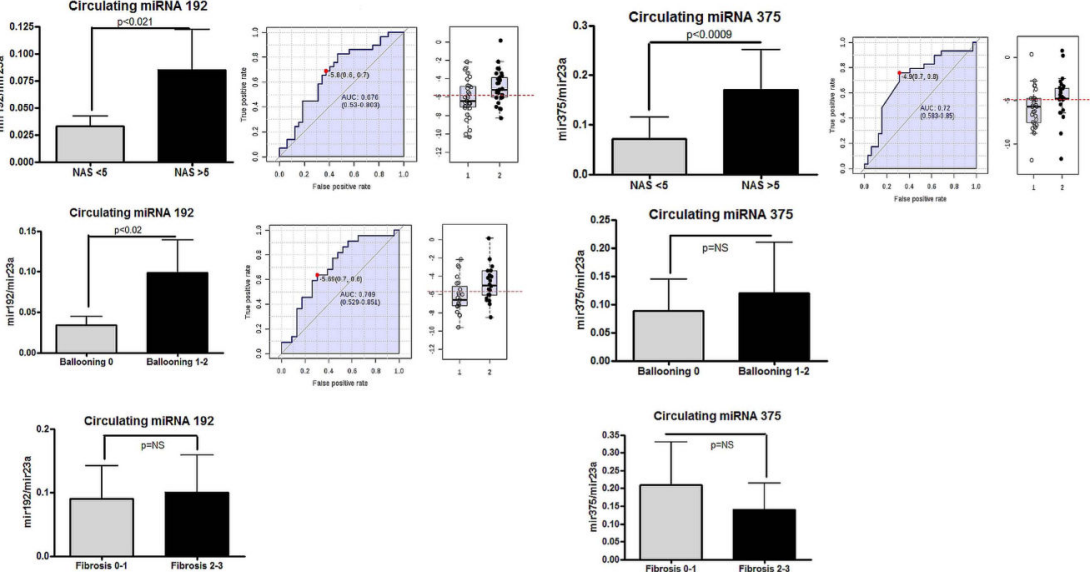

Figure 2.

Validation study of selected circulating miRNAs in non-alcoholic fatty liver disease (NAFLD) patients and control subjects, and diagnostic accuracy in predicting histological disease severity. Circulating levels of miR-122, miR-192 and miR-375 are expressed relative to miR-23a. NASH, non-alcoholic steatohepatitis; SS, simple steatosis. The criteria for evaluating the severity of fatty liver infiltration, NAS and hepatocellular ballooning are described in the Methods Section. The receiver operating characteristic (ROC) curves with the corresponding area under the ROC curves for different histological features in NAFLD patients are shown. 

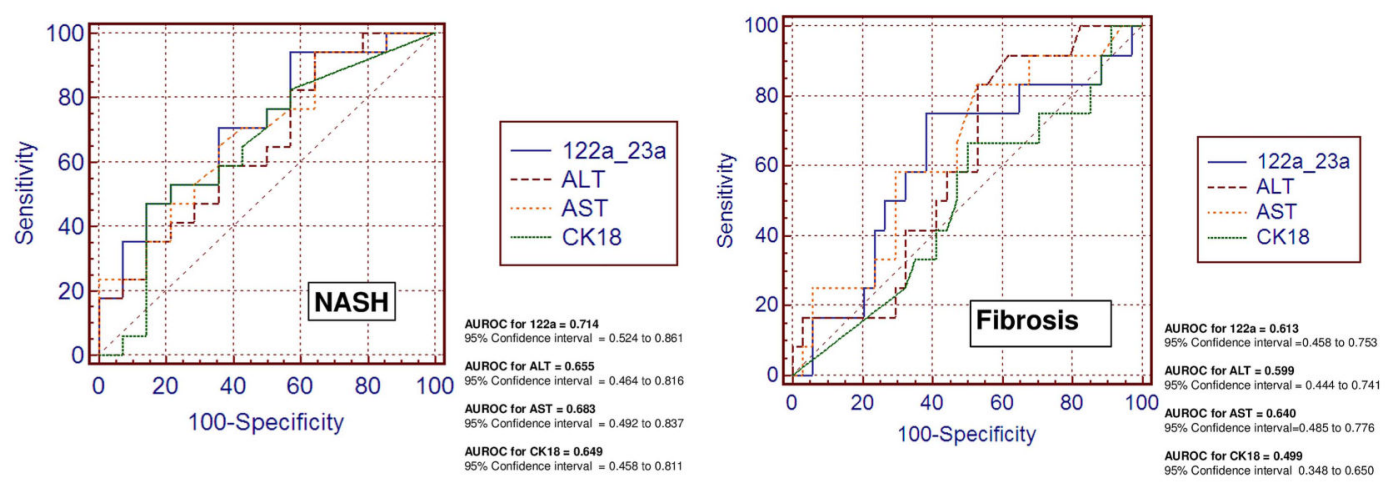

Figure 3.

Comparison of the diagnostic accuracy of circulating miR-122 and classic serum biomarkers in predicting disease severity. Receiver operating characteristic (ROC) curves with corresponding area under the ROC curves for comparing the ability of miR-122 and serum disease biomarkers, such as liver enzymes (serum alanine and aspartate aminotransferase (ALT and AST)) and caspase-generated CK-18 fragments, to distinguish the severity of nonalcoholic fatty liver disease (non-alcoholic steatohepatitis and liver fibrosis) are shown. 

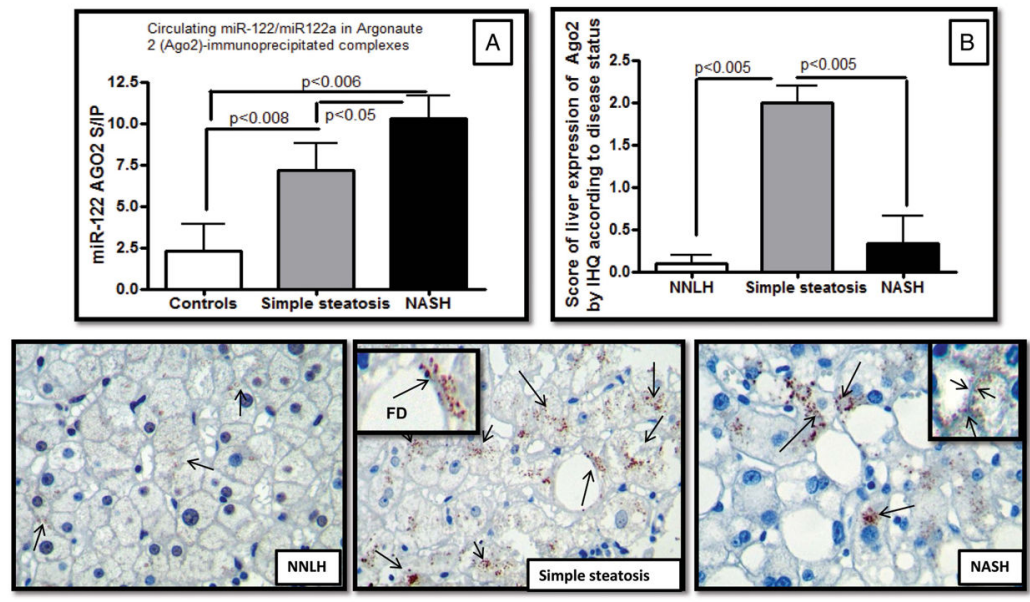

Figure 4.

Analysis of circulating miR-122 in supernatant and miR-122 in argonaute2 (Ago2)immunoprecipitated complexes in patients with non-alcoholic fatty liver disease (NAFLD). (A) Measurement of circulating expression of miR-122 in serum of controls and NAFLD patients after immunoprecipitation of serum with Ago2 (miR-122 in supernatant/miR-122 coimmunoprecipitated with anti-Ago2 antibody). (B) The scores on liver protein expression of Ago2 evaluated by immunohistochemistry with the use of a monoclonal antihuman antibody according to disease status. Results are expressed as mean $\pm \mathrm{SE}$ and median (range). The $\mathrm{p}$ value stands for statistical significance in a Kruskal-Wallis test. Bottom, a representative liver expression pattern of Ago2 evaluated by immunohistochemistry in patients with NAFLD and in control liver (black arrowheads show positive staining). Ago2 immunoreactivity was examined by light microscopy of liver sections. Anti-Ago2 antibody immunostaining was significantly observed in the cytoplasm of hepatocytes and was primarily but not exclusively restricted to lipid-laden ones (inset: fat droplet, FD) and the edge of the wall of hepatocytes (inset) showing a granular pattern. Lobular inflammatory infiltrate showed negative immunoreactivity to Ago2. Counterstaining was done with haematoxylin. Original magnification: 400x. 


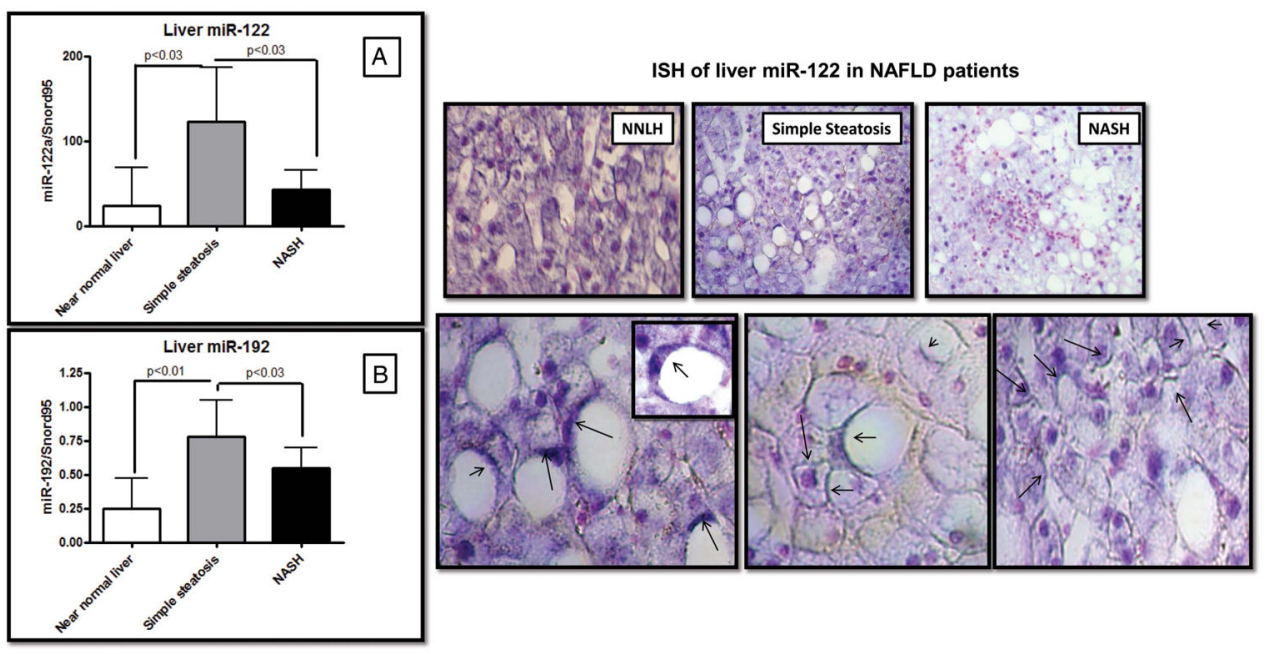

Figure 5.

Liver expression of candidate miRNAs and localisation by in situ hybridisation (ISH). Right, liver expression of miR-122 (A) and miR-192 (B) in non-alcoholic fatty liver disease (NAFLD) patients and subjects with near-normal liver histology. Left, a representative liver miR-122 ISH analysis in cases and controls, showing positive staining in blue/purple and nuclei staining in pink. Overall, the staining was particularly localised around the lipid droplets (black arrows), regardless of the NAFLD histological severity, and at the edge of the wall of hepatocytes. Original magnification: $400 \times$. 

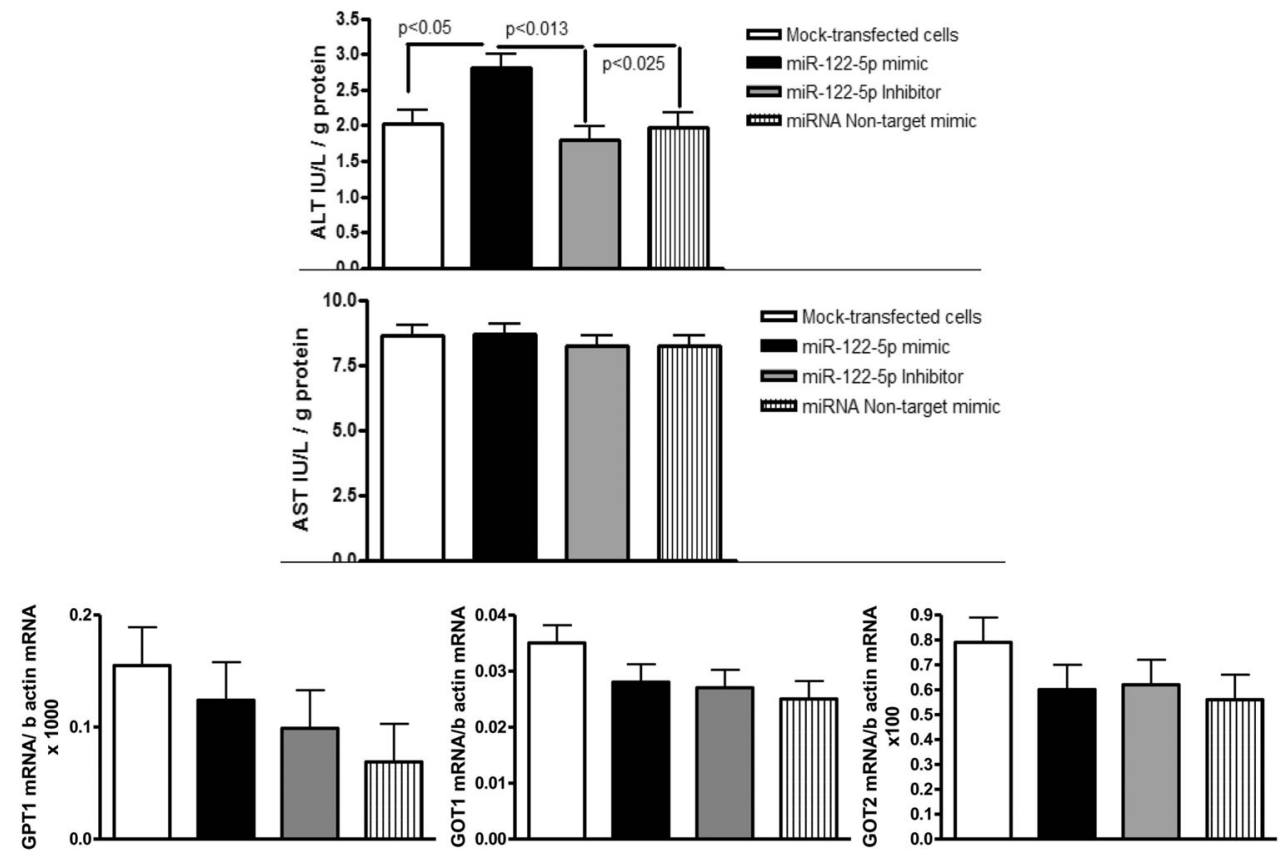

Figure 6.

In vitro exploration of the effect of miR-122 on alanine (ALT) and aspartate aminotransaminases (AST). Huh7 human hepatoma cells were transfected with a synthetic miR-122 and a miR-122 inhibitor to explore the role of miR-122 in the liver messenger RNA (mRNA) expression and enzymatic activity of liver transaminases. The liver expression of GPT1, GOT1 and GOT2 mRNA was unchanged during both miR-122 overexpression and silencing, whereas the ALT (GPT) enzymatic activity in cell lysates showed a significant increase when cells were transfected with the miR-122 mimic. GPT1, cytosolic alanine aminotransaminase 1; GOT1, glutamic-oxaloacetic transaminase 1, soluble; GOT2, glutamic-oxaloacetic transaminase 2, mitochondrial. 


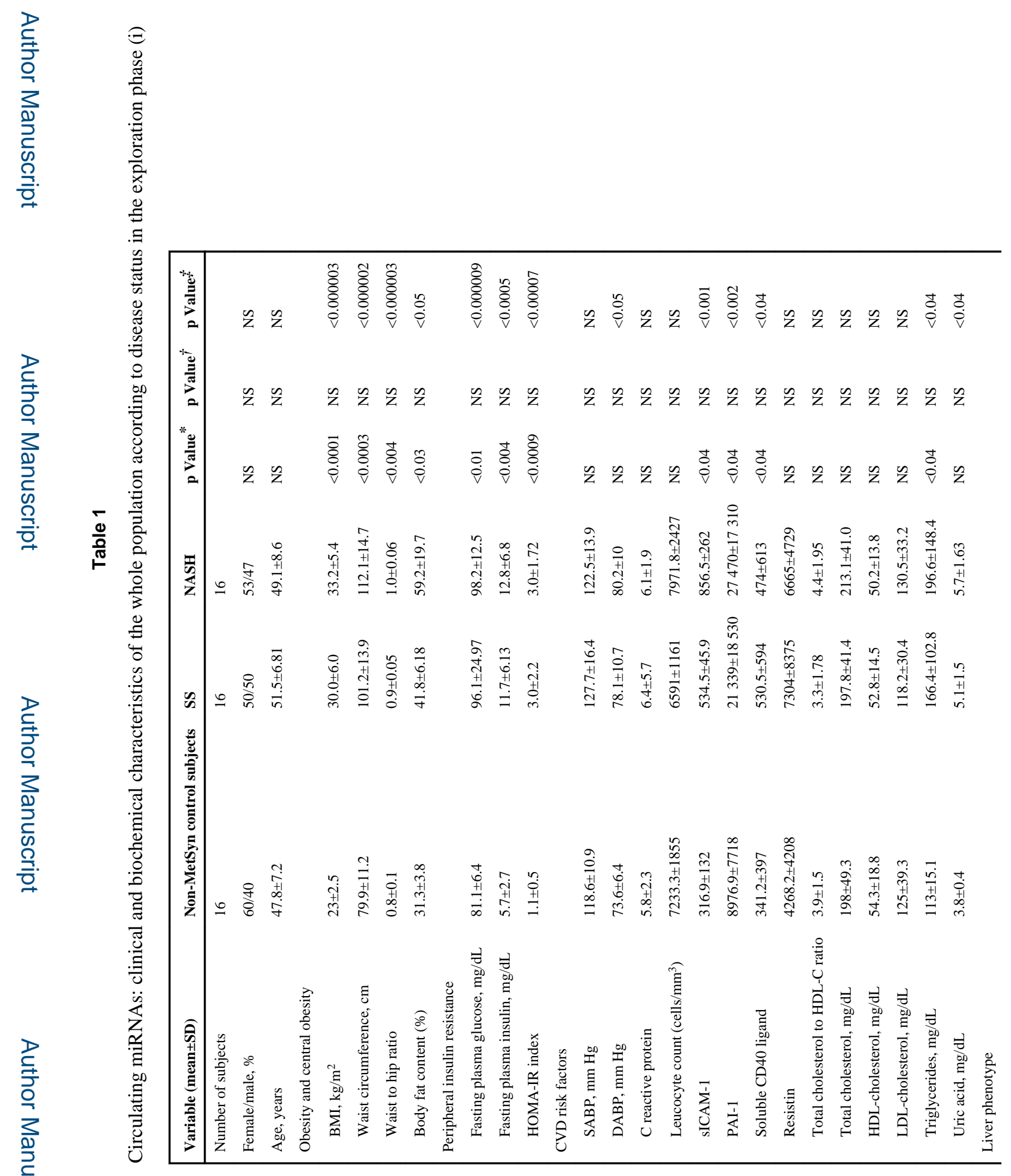

Gut. Author manuscript; available in PMC 2015 May 01. 


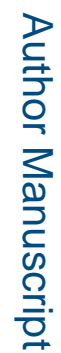

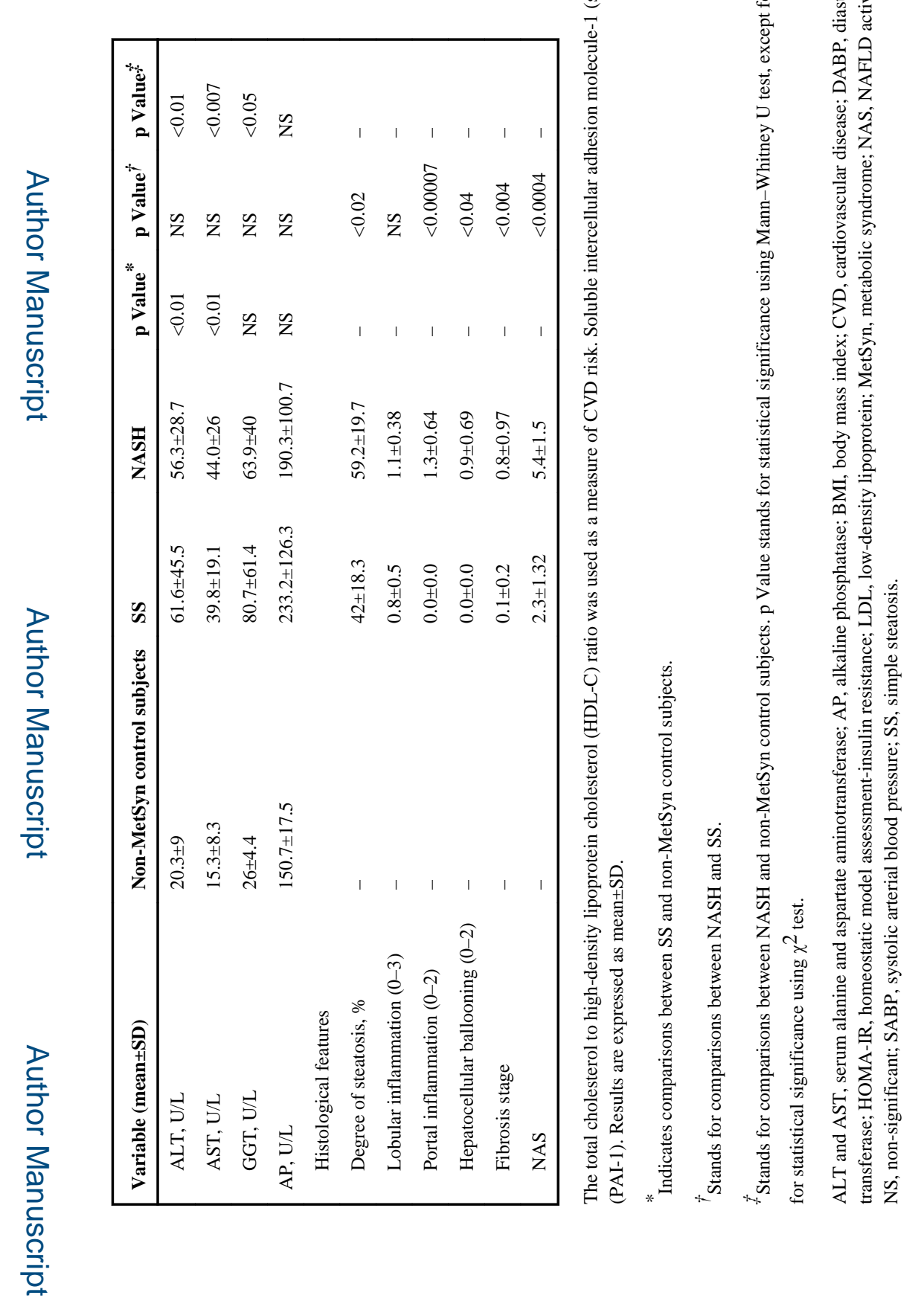

Gut. Author manuscript; available in PMC 2015 May 01. 


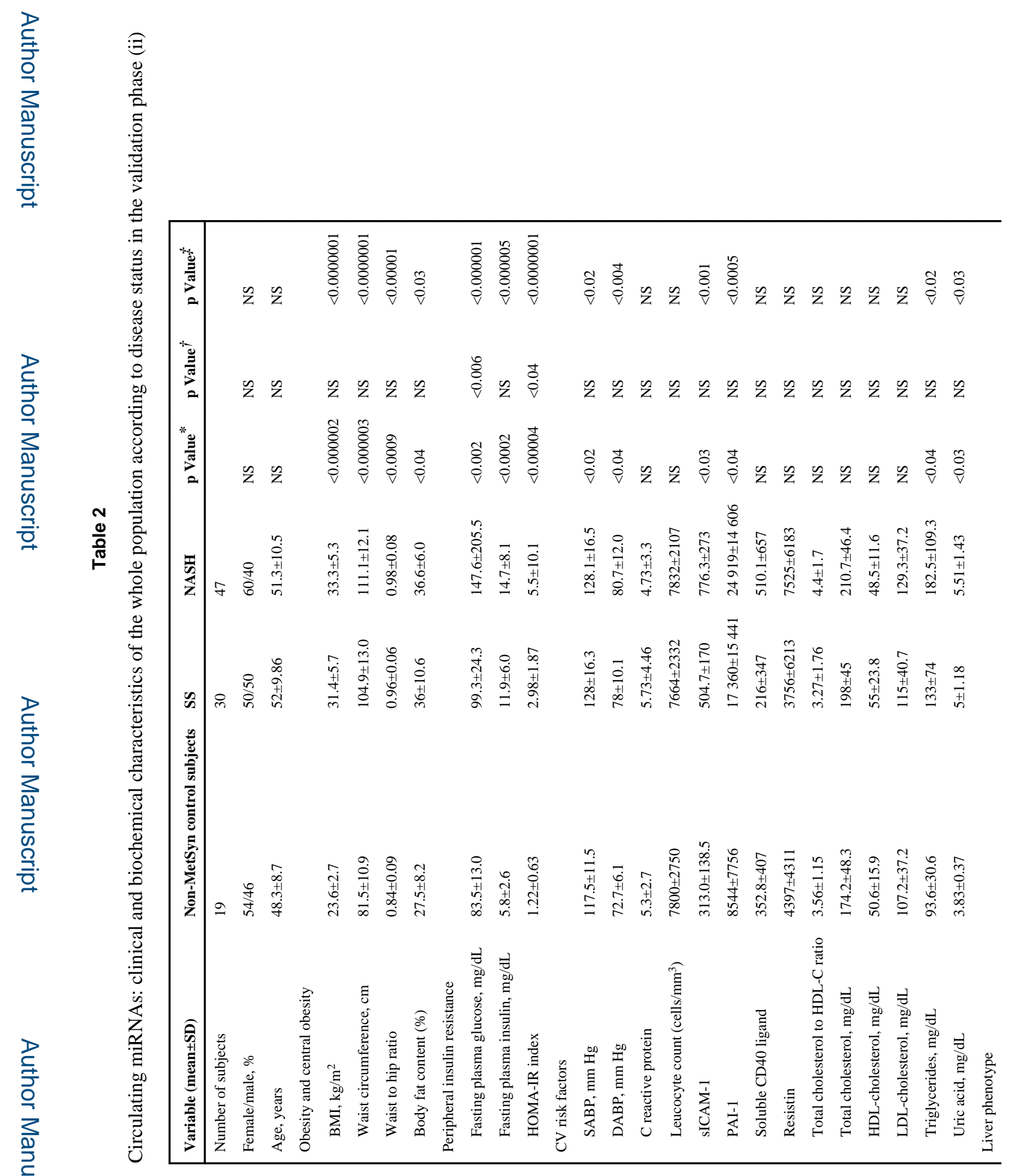

Gut. Author manuscript; available in PMC 2015 May 01. 


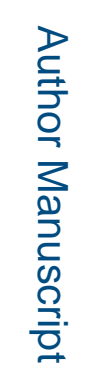

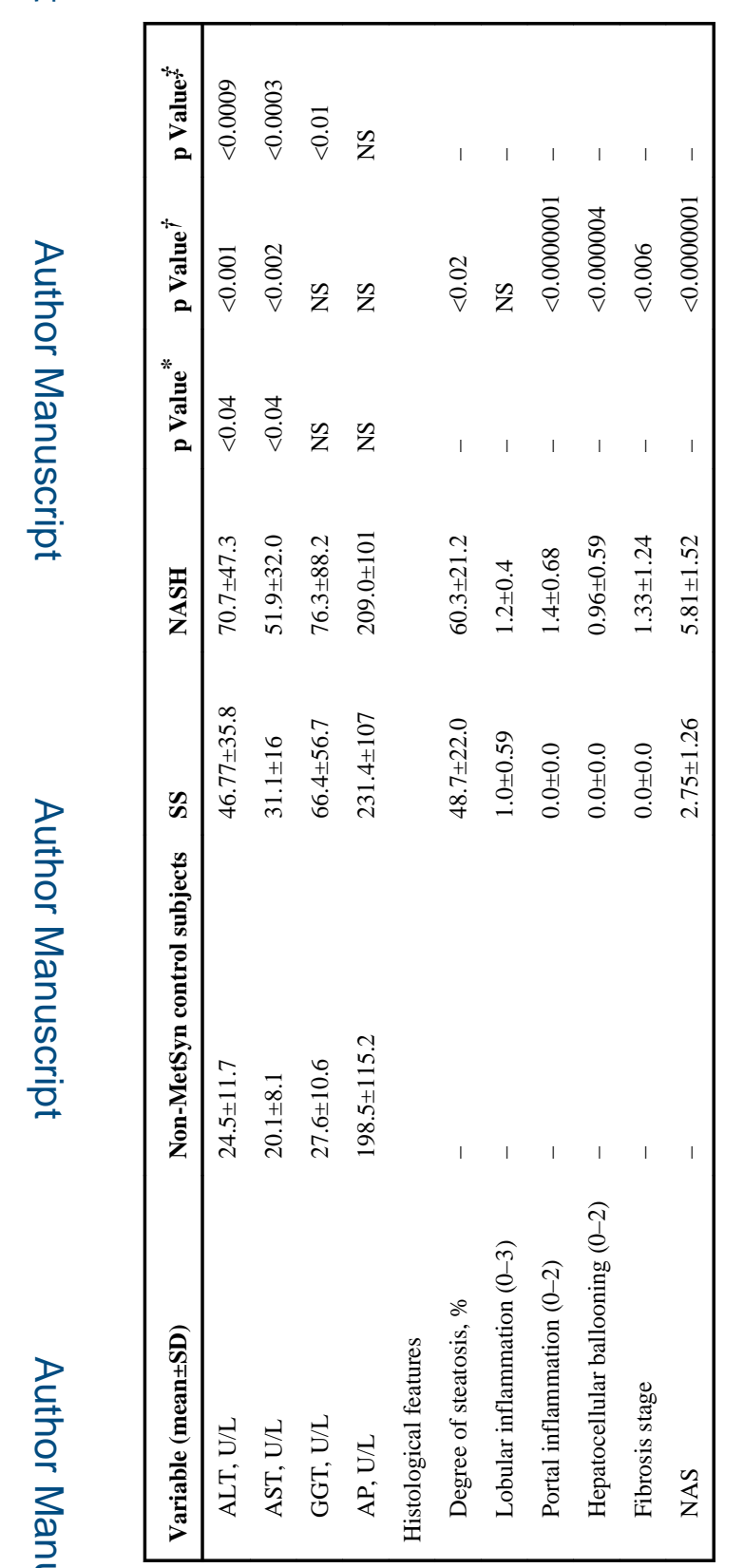

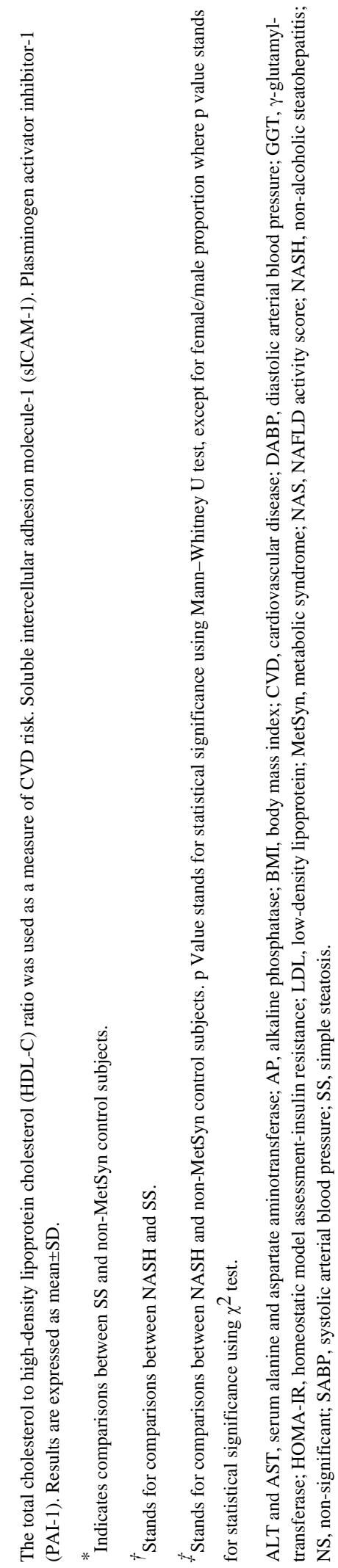

Gut. Author manuscript; available in PMC 2015 May 01. 


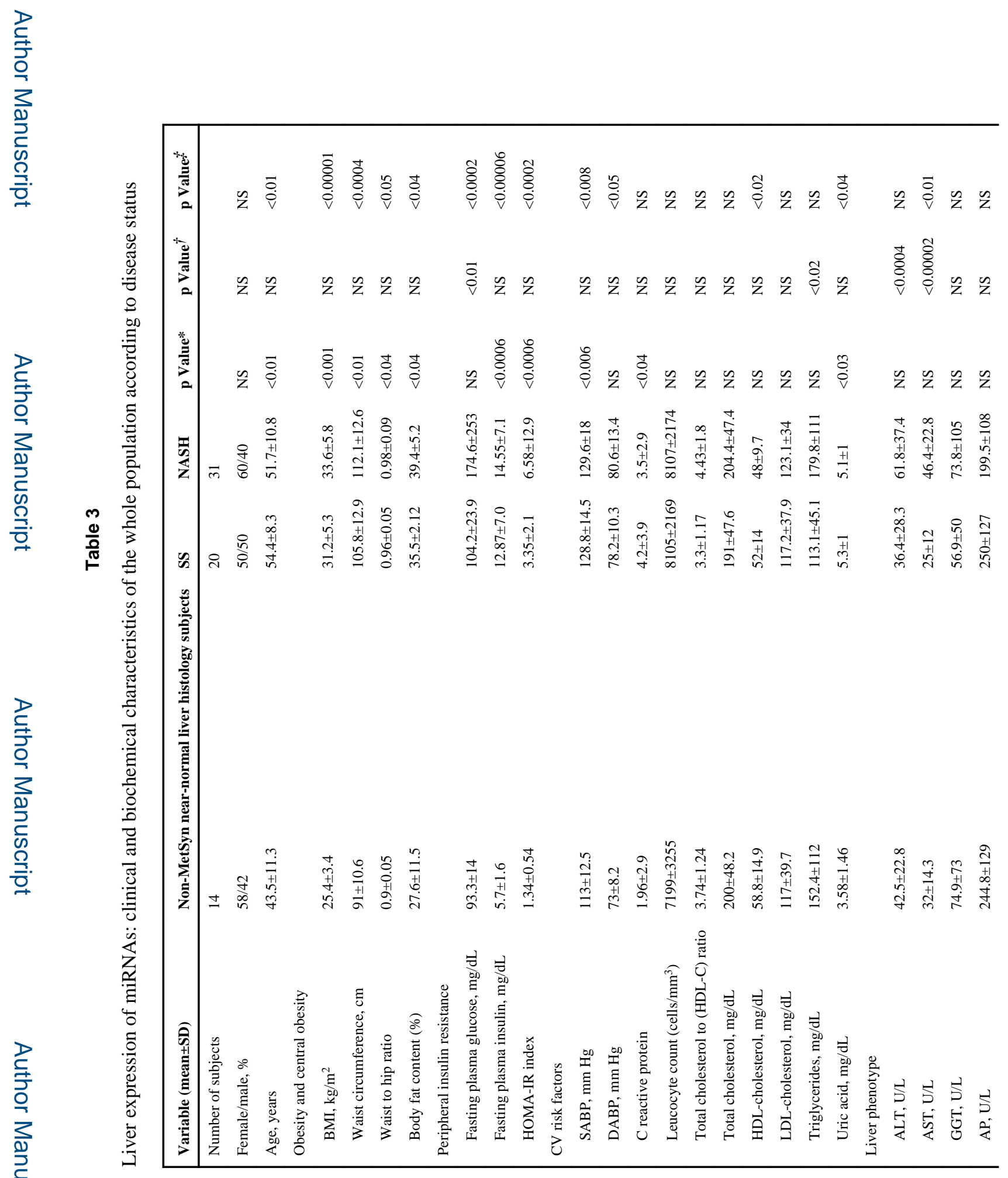

Gut. Author manuscript; available in PMC 2015 May 01. 


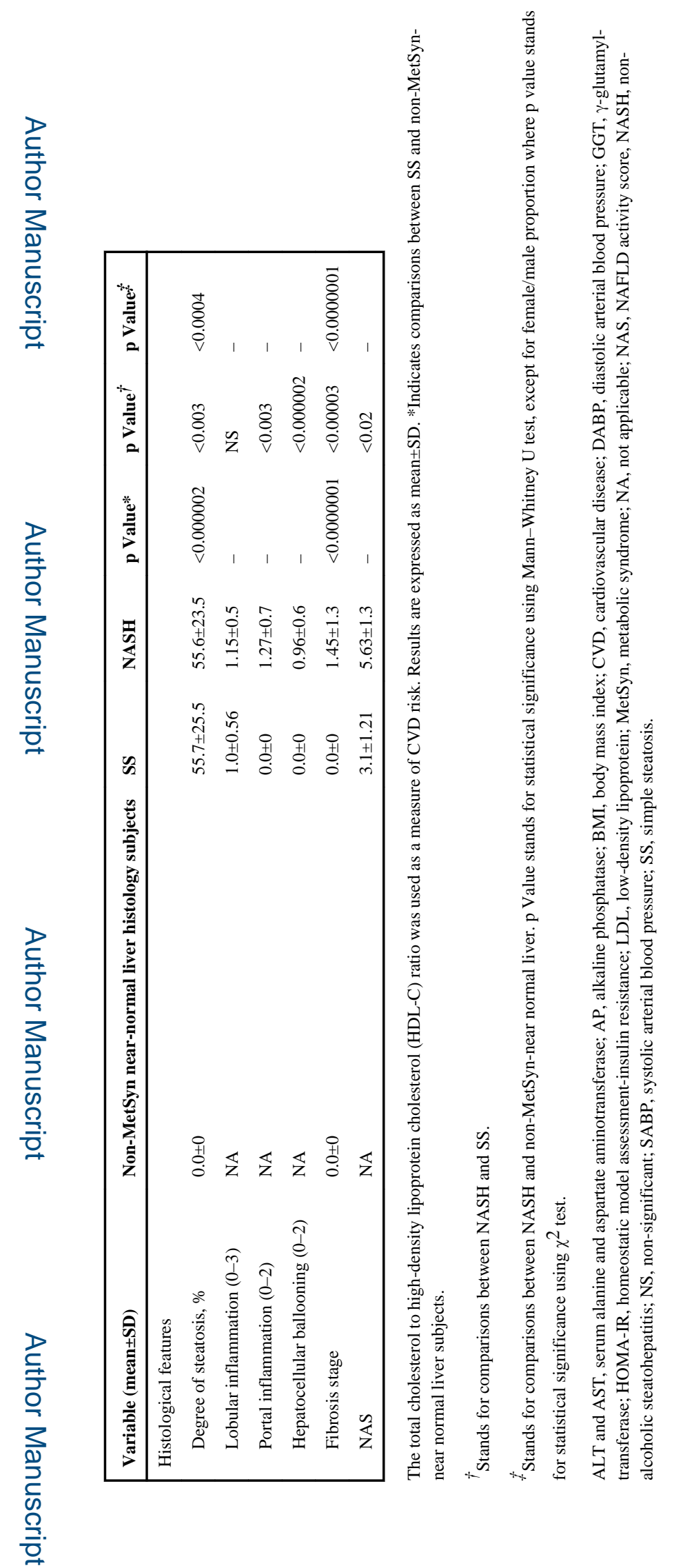

Gut. Author manuscript; available in PMC 2015 May 01. 


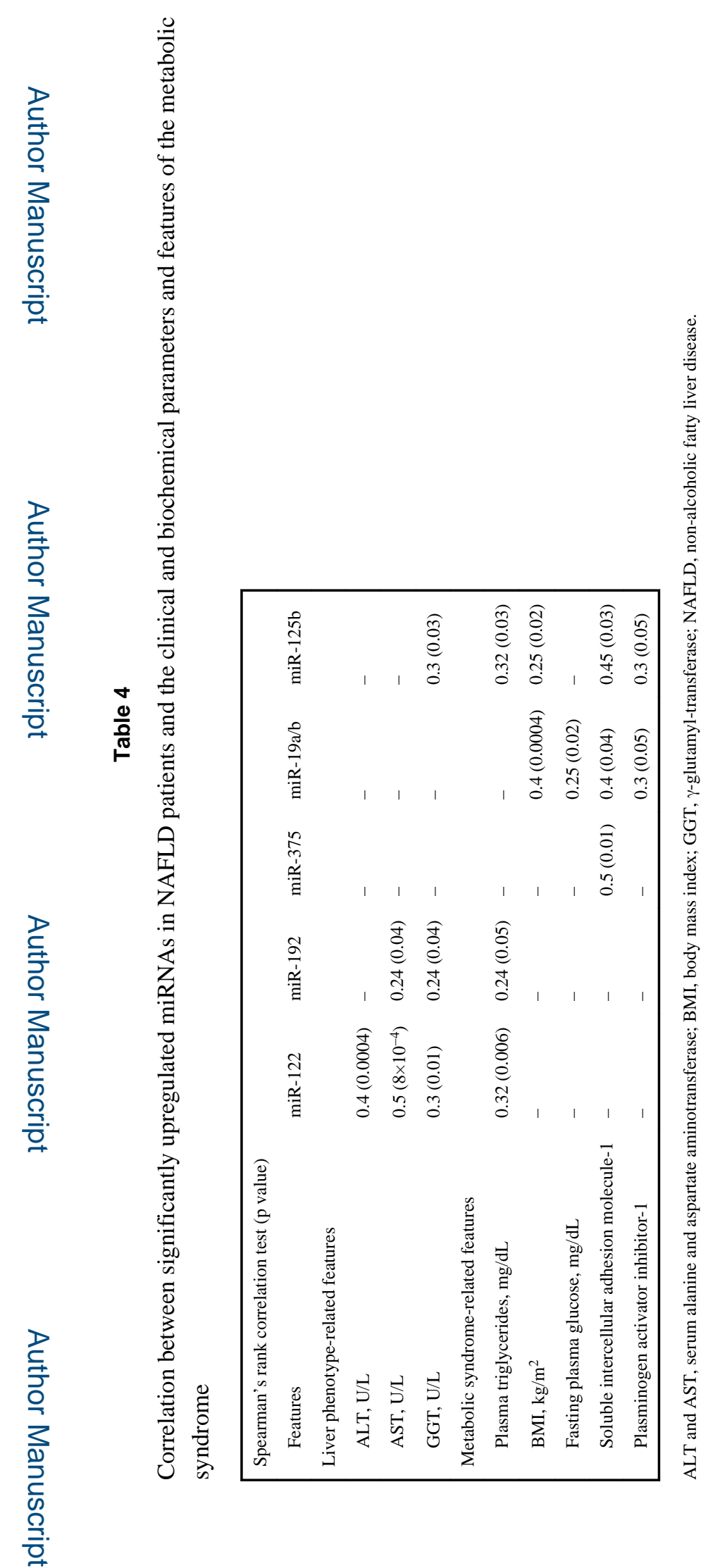

Gut. Author manuscript; available in PMC 2015 May 01. 